\title{
Correlated-electron theory of strongly anisotropic metamagnets
}

\author{
K. Held* and M. Ulmke \\ Theoretische Physik III, Universität Augsburg, D-86135 Augsburg, Germany \\ N. Blümer ${ }^{\dagger}$ \\ Department of Physics, University of Illinois at Urbana-Champaign, Urbana, Illinois 61801-3080 \\ D. Vollhardt \\ Theoretische Physik III, Universität Augsburg, D-86135 Augsburg, Germany
}

(Received 25 April 1997)

\begin{abstract}
Metamagnetic transitions in strongly anisotropic antiferromagnets are investigated within a quantum mechanical theory of correlated electrons. We employ the Hubbard model with staggered magnetization $\mathbf{m}_{\text {st }}$ along an easy axis $\mathbf{e}$ in a magnetic field $\mathbf{H} \| \mathbf{e}$. On the basis of the dynamical mean-field theory (DMFT) this model is studied both analytically and numerically. At intermediate couplings the self-consistent DMFT equations, which become exact in the limit of a large coordination number, are solved by finite temperature quantum Monte Carlo techniques. The temperature and magnetic-field dependence of the homogeneous and staggered magnetization are calculated and the magnetic phase diagram is constructed. At half filling the metamagnetic transitions are found to change from first order at low temperatures to second order near the Néel temperature, implying the existence of a multicritical point. Doping with holes or electrons has a strong effect: the system becomes metallic, the electronic compressibility increases, and the critical temperatures and fields decrease. These results are related to known properties of insulating metamagnets such as $\mathrm{FeBr}_{2}$, metallic metamagnets such as UPdGe, and the giant and colossal magnetoresistance found in a number of magnetic bulk systems. [S0163-1829(97)03446-2]
\end{abstract}

\section{INTRODUCTION}

The concept of antiferromagnetic order was proposed independently by Néel $^{1}$ and Landau. ${ }^{2}$ Both sought to explain the, at that time, puzzling low temperature behavior of the magnetic susceptibility of certain materials: of metals such as $\mathrm{Cr}$ and $\mathrm{Mn}$ in the case of Néel, and of insulators with a layered structure such as the chlorides of $\mathrm{Cr}$, bivalent $\mathrm{Fe}, \mathrm{Co}$, and $\mathrm{Ni}$ in the case of Landau. While Néel correctly suggested the existence of interpenetrating sublattices in $\mathrm{Cr}$ and $\mathrm{Mn}$ with opposite magnetization, ${ }^{1}$ Landau equally correctly predicted the existence of stacks of ferromagnetically ordered layers whose magnetization alternates from layer to layer. ${ }^{2}$ In both cases the total spontaneous magnetization adds up to zero. Assuming the interlayer coupling to be weak, Landau ${ }^{2}$ argued that a relatively small magnetic field would be sufficient to modify the mutual orientation of the moments in each layer. This leads to deviations from the linear dependence of the total moment on the field, i.e., to an anomalous increase of the susceptibility, and finally - at high fields - to a saturation of the magnetization. Such a behavior was indeed observed by Becquerel and van den $\mathrm{Handel}^{3}$ in the carbonate of $\mathrm{Fe}$ and $\mathrm{Mg}$ at low temperatures. Not being aware of Landau's or Néel's work they could not explain their observation in terms of ferro- and paramagnetism, and therefore suggested for it the name metamagnetism. ${ }^{4}$

These carbonates and other systems like $\mathrm{FeCl}_{2}$ and $\mathrm{Dy}_{3} \mathrm{Al}_{5} \mathrm{O}_{12}$ (DAG) (Ref. 5) belong to a class of insulators where the valence electrons are localized at the $\mathrm{Fe}(\mathrm{Mg})$ and Dy ions, respectively. The resulting local moments order antiferromagnetically and are constrained to lie along an easy axis e, implying a strong anisotropy such that a spin flop transition cannot occur. Under the influence of a large mag- netic field $\mathbf{H} \| \mathbf{e}$ the staggered magnetization vanishes in a phase transition, the so-called metamagnetic transition. This phase transition may be of first or second order and several of these insulating systems even show both: the first order phase transition becomes second order for temperatures $T$ higher than the tricritical temperature. At the metamagnetic transition the homogeneous susceptibility has a discontinuity. Apart from the materials mentioned above, there are also strongly anisotropic metamagnets that are conducting, e.g., uranium-based mixed systems, ${ }^{5,6} \mathrm{SmMn}_{2} \mathrm{Ge}_{2},{ }^{7}$ and probably also $\mathrm{TbRh}_{2-x} \mathrm{Ir}_{x} \mathrm{Si}_{2}{ }^{8}$ We note that the term metamagnetic transition has been adopted also to those systems where the homogeneous susceptibility as a function of the magnetic field shows no discontinuity but only a maximum. With this definition many different systems become metamagnets. ${ }^{9}$

Theoretical investigations of metamagnetic transitions and tricritical points, began with the work of Landau, who described multicritical behavior within his phenomenological theory of phase transitions. ${ }^{10}$ Clearly, a genuine understanding of the origin of tricritical points, etc. requires investigations on a more microscopic level. In the case of strongly anisotropic metamagnets those investigations where sofar restricted to the insulating systems, such as $\mathrm{FeCl}_{2}$. They are usually based on the Ising model with antiferromagnetic and ferromagnetic interactions. ${ }^{11}$ In mean-field theory Kincaid and Cohen ${ }^{12}$ showed that the tricritical point (TCP) may separate into a critical endpoint $(\mathrm{CE})$ and a bicritical endpoint (BCE) (see Fig. 1). Numerical calculations ${ }^{13-16}$ beyond mean-field theory do not find this behavior. Instead Selke ${ }^{15}$ observed anomalously strong noncritical fluctuations in the vicinity of a BCE. Experimentally a scenario similar to that shown in Fig. 1(b) was reported for $\mathrm{FeBr}_{2} \cdot{ }^{17}$ Here magnetization measurements ${ }^{18}$ merely showed weak anomalies 

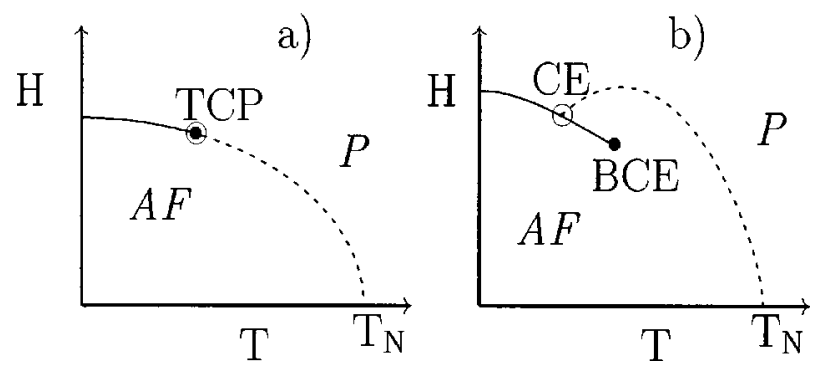

FIG. 1. Schematic $H-T$ phase diagram of (a) a typical Ising-type metamagnet and (b) the Ising model in mean-field theory with only weak ferromagnetic interaction. Solid lines: first order transition, broken lines: second order transition; AF: antiferromagnetic phase, $P$ : paramagnetic phase.

within the antiferromagnetic phase, while recent experiments on the specific heat, ${ }^{19}$ neutron scattering, ${ }^{20}$ and Faraday contrast microscopy ${ }^{21}$ seem to provide evidence for a critical line $\mathrm{CE} \leftrightarrow \mathrm{BCE}$.

The conducting systems such as the uranium-based mixed systems ${ }^{5,6}$ were hitherto described by the semiphenomenological theories of Wohlfarth and Rhodes ${ }^{22}$ for metamagnetic phase transitions in paramagnets, and of Moriya and Usami ${ }^{23}$ for the coexistence of ferro- and antiferromagnetism in itinerant electron systems. A microscopic understanding of metamagnetism in these systems requires a fully quantum mechanical treatment of itinerant, correlated electrons. Such an investigation, based on a Hubbard model with easy axis in a magnetic field, was started by us recently for the insulating systems, and some results were reported in Ref. 24 . We will now present details of our calculations and further results for the insulating case. Since the above model is the simplest microscopic model for insulating and metallic, spin-localized and bandlike antiferromagnets with easy axis in a magnetic field, we will now also investigate the metallic regime (finite doping) and consider the possibility of insulator-metal transitions in a magnetic field.

In Sec. II the underlying model, the Hubbard model with easy axis, is introduced and its validity is discussed. Then the dynamical mean-field equations which are used to investigate the correlation problem all the way from weak to strong coupling, as well as the quantum Monte Carlo techniques employed to solve them, are discussed in Sec. III. The results obtained for a half filled band and for finite doping are presented in Sec. IV and Sec. V, respectively. A discussion of the results in Sec. VI closes the presentation.

\section{HUBBARD MODEL WITH EASY AXIS}

The Hubbard model ${ }^{25}$ is the generic microscopic model for itinerant and localized antiferromagnetism in correlated electron systems. For nearest-neighbor hopping of electrons in the presence of a Zeeman term it has the form

$$
\hat{H}=-t \sum_{\mathrm{NN}, \sigma} \hat{c}_{i \sigma}^{\dagger} \hat{c}_{j \sigma}+U \sum_{i} \hat{n}_{i \uparrow} \hat{n}_{i \downarrow}-\sum_{i, \sigma}(\mu+\sigma H) \hat{n}_{i \sigma}
$$

where operators carry a hat. From the exact, analytic solution in dimensions $d=1$ the (paramagnetic) ground state of this model at half filling $(n=1)$ is known to exhibit metamag- netic behavior, i.e., $\partial \chi / \partial H>0$, up to saturation. ${ }^{26}$ However, this is entirely due to the convex shape of the density of states in $d=1$ and occurs even at $U=0$.

In general the Hubbard model in the form of Eq. (1) cannot describe metamagnetic behavior. The Hubbard model is isotropic in spin space and hence the antiferromagnetic phase described by it is isotropic, too. Consequently, any finite magnetic field $\mathbf{H}$ will orient the staggered magnetization $\mathbf{m}_{\mathrm{st}}$ perpendicular to itself. Real antiferromagnets are, however, never isotropic in spin space: the relativistic spin-orbit interaction $\hat{\mathbf{L}} \cdot \hat{\mathbf{S}}$, where $\hat{\mathbf{L}}$ and $\hat{\mathbf{S}}$ are operators for the orbital angular momentum and spin, respectively, transfers the anisotropy of position space (caused by the broken rotational symmetry of the crystal lattice) into spin space, producing one, or more, easy axes which constrain the spins. ${ }^{27}$ In an external magnetic field such a constraint leads to metamagnetic transitions, with or without a spin-flop depending on the strength of the spin-orbit interaction, as explained in Sec. I.

A microscopic theory of strongly anisotropic antiferromagnets should ultimately be able to take into account the orbital degeneracy of the electrons and, by including the relativistic spin-orbit interaction $\hat{\mathbf{L}} \cdot \hat{\mathbf{S}}$ in the Hamiltonian, to generate an anisotropy axis within the model itself. At present, this is technically not possible. ${ }^{28}$ Therefore we take the existence of the anisotropy axis $\mathbf{e}$ for granted: we employ the Hubbard model (1) and constrain the magnetic moments to lie along $\mathbf{e} \| \mathbf{H}$. By this approach the kinetic energy and the Coulomb interaction are treated microscopically, whereas the relativistic corrections are not. We note that the relativistic corrections are of the order of $10^{-2} \mathrm{eV}$ and are thus small compared to energies of the order of $1 \mathrm{eV}$ for kinetic and Coulomb energy. Therefore the existence of $\mathbf{e}$ and the correlation physics described by the Hubbard model (1) are quite unrelated. This justifies our approach where the existence of e is a priori assumed. ${ }^{29}$

\section{DYNAMICAL MEAN-FIELD THEORY $(d \rightarrow \infty)$}

For classical spin models (e.g., the Ising model) it is well known that the Weiss molecular field theory becomes exact in the limit of high spatial dimensions $(d=\infty)$. For lattice electrons this limit was introduced only recently. ${ }^{30}$ With the proper scaling of the hopping element in Eq. (1), $t=t * / \sqrt{Z}$ ( $Z=$ number of nearest neighbors), it leads to a quantum mechanical dynamical mean-field theory (DMFT); for reviews see (Refs. 31 and 32) The interacting lattice model then reduces to a self-consistent single site problem of electrons in an effective medium, ${ }^{33}$ which may be described by a complex, frequency dependent (i.e., dynamical) self-energy $\Sigma^{\sigma}(\omega)$. This problem is, in fact, equivalent to an Anderson impurity model complemented by a self-consistency condition. ${ }^{34-36}$

There are two limits in which the DMFT recovers wellknown static mean-field theories:

(1) Weak coupling: In this situation the effective medium may be approximated by a static field which is generated by the averaged densities of the electrons. This leads to the Hartree-Fock approximation, e.g., $\Sigma^{\sigma}(\omega)=U n_{-\sigma}$ in the homogeneous case, which is expected to give the qualitatively 
correct behavior at weak coupling. The averaged densities $n_{\sigma}$ have to be determined self-consistently.

(2) Strong coupling at half filling: Here model (1) can be mapped onto the antiferromagnetic spin 1/2 Heisenberg model for which the limit $d=\infty$ becomes equivalent to the Weiss molecular field theory.

The results obtained in these two limits will be presented, and compared to the results for intermediate coupling, in Sec. IV.

During the past few years the DMFT has provided valuable insight into the physics of the Mott-Hubbard transition ${ }^{32,35,37}$ and of transport properties of strongly correlated electron systems. ${ }^{38}$ The effect of the magnetic field $H$ in Eq. (1) was previously studied at half filling. ${ }^{39-41}$ In particular, Laloux et al. ${ }^{39}$ investigated the magnetization behavior of the paramagnetic phase. They found that, at strong enough interaction $U$, the magnetic field can trigger a metalinsulator transition. Since on the insulating side the magnetization of the local moments is enhanced the metal-insulator transition shows up as a discontinuity in the susceptibility. Hence this transition is also referred to as a "metamagnetic transition." ${ }^{42}$ Giesekus and Brandt ${ }^{41}$ took into account also the AF order. They considered the isotropic case where the field orients the staggered magnetization perpendicular to itself, such that a metamagnetic transition cannot occur.

To investigate the metamagnetic phase transition from an antiferromagnet to a paramagnet we consider a bipartite $(A-B)$ lattice and allow for symmetry breaking with respect to spin $\sigma \in\{\uparrow, \downarrow\}=\{+,-\}$ and sublattice $\alpha \in\{A, B\}$ $=\{+,-\}$. The self-energy $\Sigma_{\alpha n}^{\sigma} \equiv \Sigma_{\alpha}^{\sigma}\left(i \omega_{n}\right)$, with Matsubara frequencies $\omega_{n}=\pi T(2 n+1), n=0, \pm 1, \pm 2, \ldots$ (using the convention $\hbar \equiv k_{B} \equiv 1$ ), and the Green function $G_{\alpha n}^{\sigma}$ are determined self-consistently by two sets of coupled equations: ${ }^{33,34}$

$$
\begin{gathered}
G_{\alpha n}^{\sigma}=\int_{-\infty}^{\infty} d \epsilon \frac{N^{0}(\epsilon)}{z_{\alpha n}^{\sigma}-\epsilon^{2} / z_{-\alpha n}^{\sigma}}, \\
G_{\alpha n}^{\sigma}=-\left\langle\psi_{\sigma n} \psi_{\sigma n}^{*}\right\rangle_{A_{\alpha}} .
\end{gathered}
$$

Here $z_{\alpha n}^{\sigma}=i \omega_{n}+\mu-\Sigma_{\alpha n}^{\sigma}$, and the thermal average of some operator $\hat{O}$ in Eq. (3) is defined as a functional integral over the Grassmann variables $\psi, \psi^{*}$, with

$$
\langle\hat{O}\rangle_{A_{\alpha}}=\frac{1}{Z_{\alpha}} \int \mathcal{D}[\psi] \mathcal{D}\left[\psi^{*}\right] O\left[\psi, \psi^{*}\right] e^{A_{\alpha}\left[\psi, \psi^{*}, \Sigma, G\right]},
$$

in terms of the single site action

$$
\begin{aligned}
A_{\alpha}= & \sum_{\sigma, n} \psi_{\sigma n}^{*}\left[\left(G_{\alpha n}^{\sigma}\right)^{-1}+\Sigma_{\alpha n}^{\sigma}\right] \psi_{\sigma n} \\
& -U \int_{0}^{\beta} d \tau \psi_{\uparrow}^{*}(\tau) \psi_{\uparrow}(\tau) \psi_{\downarrow}^{*}(\tau) \psi_{\downarrow}(\tau),
\end{aligned}
$$

where $Z_{\alpha}$ is the partition function, and $N^{0}(\epsilon)$ is the density of states (DOS) of the non interacting electrons. As the results do not much depend on its precise form we choose a half-elliptic DOS $N^{0}(\epsilon)=\left[\left(2 t^{*}\right)^{2}-\epsilon^{2}\right]^{1 / 2} /\left(2 \pi t^{* 2}\right)$. The constraint $\mathbf{m}_{\text {st }} \| \mathbf{H}$ is enforced by setting the off-diagonal (in spin space) elements of the Green function equal to zero:
$G_{\alpha}^{\downarrow \uparrow}=-\left\langle\psi_{\alpha}^{\downarrow} \psi_{\alpha}^{\uparrow *}\right\rangle \equiv 0$. From now on $t^{*} \equiv 1$ will set our energy scale, ${ }^{43}$ i.e., the total bandwidth of $N^{0}(\epsilon)$ is equal to 4 . The Dyson equation (2) introduces the lattice into the problem. It couples $A$ and $B$ sublattices and can be solved by a simple integration, even analytically for the above DOS. The functional integral (3) however is highly non-trivial since it couples all Matsubara frequencies. Georges and Kotliar $^{34}$ and Jarrell ${ }^{35}$ realized that the action (5) is equivalent to that of an Anderson impurity model, and can therefore be treated by standard techniques developed for this model. Here we employ a finite temperature, auxiliary field quantum Monte Carlo (QMC) method. ${ }^{44,45}$ In this approach the electronelectron interaction is formally replaced by an interaction of independent electrons with a dynamical, auxiliary field of Ising-type spins. To this end the interval $[0, \beta]$ is discretized into $\Lambda$ steps of size $\Delta \tau=\beta / \Lambda$. Equivalently, there is a high energy cutoff of Matsubara frequencies, i.e., $\left|\omega_{n}\right|$ $=\pi T|2 n+1|<\pi / \Delta \tau, n=-\Lambda / 2, \ldots, \Lambda / 2-1$. All quantities have to be extrapolated to $\Delta \tau \rightarrow 0$. The computer time grows like $\Lambda^{3} \propto \beta^{3}$, restricting $\Lambda$ to values below $\sim 150$ and $\beta \leqslant 50 \ldots 70$ on present supercomputers. For small $\Lambda$ $(\Lambda \leqslant 20)$ one can perform a full enumeration (instead of the Monte Carlo sampling) of all $2^{\Lambda}$ possible configurations of the auxiliary field. We never encountered a minus-sign problem, hence no further approximations (like the "fixed-node" method) were necessary.

The self-consistency is obtained iteratively as follows: the Green function $G$ (omitting indices) is calculated from some initial self-energy, e.g., $\Sigma \equiv 0$, by the Dyson equation (2). Now the new Green function $G_{\text {new }}$ is determined by solving Eq. (3) with the QMC method. Finally, the calculation of the new self-energy $\Sigma_{\text {new }}=\Sigma-G_{\text {new }}^{-1}+G^{-1}$ completes one iteration. To improve convergence in the symmetry broken case $G_{A}$ and $G_{B}$ are updated by the Dyson equation (2) after every QMC simulation for one sublattice [Eq. (3)]. In the symmetry broken phases, typically $10-20$ iterations with $20000 \mathrm{MC}$ sweeps are necessary to obtain a convergence of $\sim 10^{-3}$ in $\Sigma\left(i \omega_{n}\right)$. The calculation of a magnetization curve at $\beta=50$ takes about $100 \mathrm{~h}$ on a Cray-Y-MP. Close to a phase transition the convergence is much slower and the statistical errors are larger due to strong fluctuations, in particular in the case of a second order phase transition. These effects limit the accuracy in the determination of the critical values of the model parameters, e.g., the critical magnetic field (see Sec. IV C). At large $U$ values $(U>4)$ the Monte Carlo sampling becomes more and more inefficient due to "sticking" problems, i.e., there are two (or more) minima in the free energy and the single spin-flip algorithm is no longer able to transfer between them.

From the resulting Green functions we calculate the densities and the homogeneous and antiferromagnetic magnetization:

$$
\begin{gathered}
n_{\alpha \sigma}=1+T \sum_{n} G_{\alpha n}^{\sigma}, \\
m=\frac{1}{2} \sum_{\alpha, \sigma} \sigma n_{\alpha \sigma}, \\
m_{\mathrm{st}}=\frac{1}{2} \sum_{\alpha, \sigma} \alpha \sigma n_{\alpha \sigma} .
\end{gathered}
$$


Since we are interested in the question whether the system is insulating or metallic we also determine the electronic compressibility $\kappa_{e} \equiv \partial n / \partial \mu$. It was calculated both by numerical differentiation of $n(\mu)$ and from the two-particle correlation functions (see Appendix A). Both results agree within the statistical errors: however, the latter method is much more time consuming.

\section{RESULTS FOR HALF FILLING}

\section{A. Weak coupling}

For weak coupling $(U \ll t)$ we expect the Hartree-Fock approximation to give an, at least qualitatively, ${ }^{46}$ correct picture of the metamagnetic phase transitions, especially of their order. Within this static mean-field theory electronic correlations are neglected and the interaction is decoupled as

$$
\hat{n}_{i \sigma} \hat{n}_{i-\sigma} \stackrel{\mathrm{HF}}{\rightarrow} \hat{n}_{i \sigma}\left\langle\hat{n}_{i-\sigma}\right\rangle+\left\langle\hat{n}_{i \sigma}\right\rangle \hat{n}_{i-\sigma}-\left\langle\hat{n}_{i-\sigma}\right\rangle\left\langle\hat{n}_{i \sigma}\right\rangle .
$$

Note, that while for $\mathbf{e}\left\|\mathbf{m}_{\text {st }}\right\| \mathbf{m}$ the Fock term vanishes, $\left\langle\hat{c}_{i \uparrow}^{\dagger} \hat{c}_{i \downarrow}\right\rangle=0$, its presence is essential in the case $\mathbf{m}_{\mathrm{st}} \perp \mathbf{m}$ (see Appendix B). We confine our investigations to states with homogeneous sublattice magnetization as described by the ansatz,

$$
\left\langle\hat{n}_{i \in \alpha, \sigma}\right\rangle=\frac{1}{2}\left(n+\sigma m+\alpha \sigma m_{\mathrm{st}}\right) .
$$

Applying this Hartree-Fock decoupling scheme one obtains the effective, one-particle Hamiltonian,

$$
\begin{aligned}
\hat{H}_{\mathrm{HF}}= & \sum_{\mathrm{NN}, \sigma} t_{i j} \hat{c}_{i \sigma}^{\dagger} \hat{c}_{j \sigma}+\sum_{\alpha, i \in \alpha, \sigma} \frac{U}{2}\left(n-\sigma m-\sigma \alpha m_{\mathrm{st}}\right) \hat{n}_{i \sigma} \\
& -(\mu+\sigma H) \hat{n}_{i \sigma}-\frac{1}{2} \frac{U}{4} \sum_{\alpha, i \in \alpha, \sigma}\left[n^{2}-\left(m+\alpha m_{\mathrm{st}}\right)^{2}\right] .
\end{aligned}
$$

This Hamiltonian is diagonalized and the one-particle energies $\widetilde{\epsilon}_{\sigma}$ are calculated as

$$
\tilde{\epsilon}_{\sigma}=\operatorname{sgn}(\epsilon) \sqrt{\epsilon^{2}+\left(\frac{U}{2} m_{\mathrm{st}}\right)^{2}}-\sigma\left(\frac{U}{2} m+H\right),
$$

where $\operatorname{sgn}(\epsilon)$ denotes the sign of the noninteracting electron energy $\epsilon$. In the antiferromagnetic phase the DOS has a gap of width $U m_{s t}$ with square root singularities at its edge.

From the one-particle energies (10) the grand potential per lattice site ( $L$ being the number of lattice sites) is obtained directly as $\left(\tilde{\mu}=\mu-\frac{1}{2} U n\right)$

$$
\begin{aligned}
\Omega / L=-\frac{1}{\beta} \ln \mathcal{Z}= & -\frac{1}{\beta} \sum_{\sigma} \int \mathrm{d} \epsilon N^{0}(\epsilon) \ln \left(1+e^{-\beta\left(\tilde{\epsilon}_{\sigma}-\tilde{\mu}\right)}\right) \\
& +\frac{U}{4}\left(m_{\mathrm{st}}^{2}+m^{2}\right)-\frac{U n^{2}}{4} .
\end{aligned}
$$

The potential $\Omega$ has two shallow minima, one corresponding to the paramagnetic state $\left(m \neq 0, m_{\text {st }}=0\right)$ and the other to the antiferromagnetic state $\left(m \approx 0, m_{\mathrm{st}} \neq 0\right)$. By applying a sufficiently strong magnetic field to the antiferromagnetic state, the paramagnetic minimum becomes the lowest, such that a first order phase transition takes place. The reason for the occurrence of a first order phase transition is that, within the (static) Hartree-Fock approximation, the $U$ term becomes minimal for largest (static) local moments. Therefore pure antiferromagnetic and ferromagnetic order are both energetically favored. Mixed states with $m \neq 0$ and $m_{\mathrm{st}} \neq 0-$ which would occur in the case of second order phase transitionshave a small local moment, i.e., a high Hartree-Fock energy, on every second site.

To calculate the magnetization curves the self-consistent Hartree-Fock equations are obtained from the minimization conditions $\partial \Omega / \partial m_{\mathrm{st}}=0$ and $\partial \Omega / \partial m=0$ :

$$
\begin{gathered}
m_{\mathrm{st}}=\frac{U}{2} \sum_{\sigma} \int d \epsilon N^{0}(\epsilon) \frac{-m_{\mathrm{st}} \operatorname{sgn}(\boldsymbol{\epsilon})}{\sqrt{\epsilon^{2}+\left(\frac{U}{2} m_{\mathrm{st}}\right)^{2}} \frac{1}{1+e^{\beta\left(\tilde{\epsilon}_{\sigma}-\tilde{\mu}\right)}}} \\
m=\sum_{\sigma} \int d \epsilon N^{0}(\epsilon) \sigma \frac{1}{1+e^{\beta\left(\widetilde{\epsilon}_{\sigma}-\tilde{\mu}\right)}}
\end{gathered}
$$

These equations are solved numerically by iteration and integration according to Newton-Cotes rules.

Within the Hartree-Fock approximation the metamagnetic phase transition is found to be of first order for all $T$, even for $U=4$ ( $=$ bandwidth); see Figs. 2 and 3. Hence a tricritical point never occurs. In this parameter range the quantum Monte Carlo calculations, however, already show second order transitions in a broad range of temperatures (see Sec. IV C). Hence the Hartree-Fock solution can neither describe the experimental situation, where tricritical points are known to occur, nor the correct behavior of the model for intermediate values of $U$.

To estimate the anisotropy energy associated with the easy axis we compare the Hartree-Fock energies of the configurations with $\mathbf{m}_{\text {st }} \| \mathbf{m}$ and $\mathbf{m}_{\text {st }} \perp \mathbf{m}$ (for details see Appendix B). At half filling and for $U$ equal to the bandwidth, the difference between the free energy of these configurations does not exceed a few percent of the bandwidth, i.e., $O\left(10^{-2}\right.$ $\mathrm{eV})$. Then the spin orbit interaction, which can be relatively strong, $O\left(10^{-1} \mathrm{eV}\right)$, indeed leads to a strong anisotropy, i.e., an easy axis $\mathbf{e}$, along which $\mathbf{m}_{\text {st }}$ is rigidly fixed.

Metamagnetic phase transitions in itinerant, metallic systems were hitherto described by the theory of "itinerant electron metamagnetism' (IEM). In the case of an antiferromagnetic system in a magnetic field Moriya and Usami ${ }^{23}$ proposed a Landau theory with free energy,

$$
\begin{aligned}
F\left(m, m_{\mathrm{st}}\right)= & \frac{1}{2 \chi_{m}} m^{2}+\frac{1}{2 \chi_{\mathrm{st}}} m_{\mathrm{st}}^{2}+a m^{4}+a^{\prime} m_{\mathrm{st}}^{4}+b m^{2} m_{\mathrm{st}}^{2} \\
& +b^{\prime}\left(\mathbf{m} \cdot \mathbf{m}_{\mathrm{st}}\right)^{2}-H m
\end{aligned}
$$

where $\chi_{m}$ and $\chi_{\text {st }}$ are the homogeneous and staggered susceptibility, respectively, and the coefficients $a, a^{\prime}, b$, and $b^{\prime}$ are the fourth-order derivatives of the noninteracting free energy. Within the IEM theory the Coulomb interaction is treated in random phase approximation. The corresponding susceptibilities are given as 


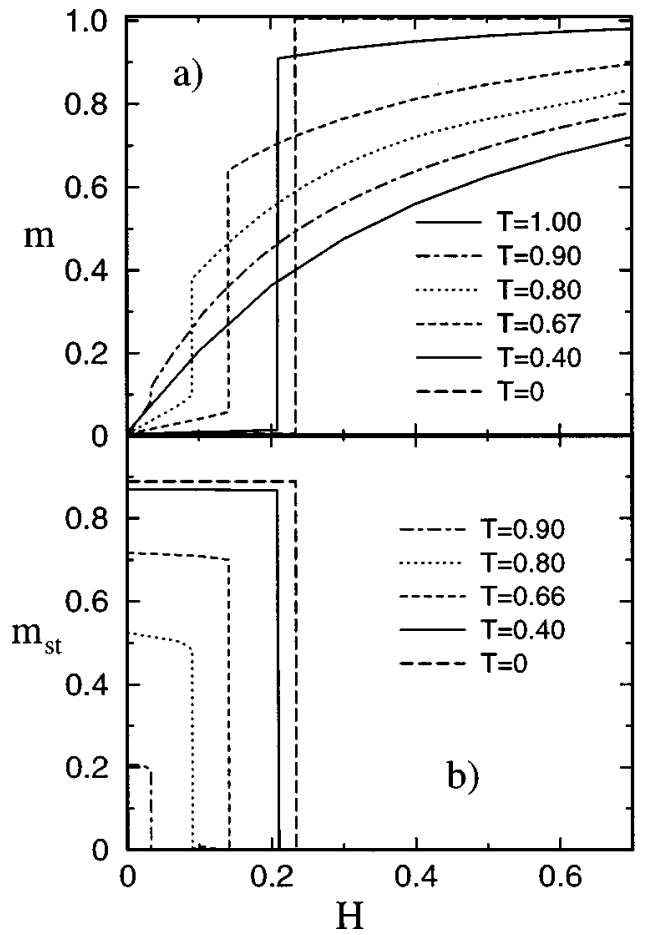

FIG. 2. (a) Magnetization $m$ vs magnetic field $H$ in HartreeFock approximation for $U=4$ at different temperatures $T$ showing metamagnetic behavior. (b) Order parameter for the metamagnetic phase transition (the staggered magnetization) $m_{\text {st }}$ vs $H$. The first order phase transition is clearly seen.

$$
\frac{1}{\chi_{m}}=\frac{1}{\chi_{m}^{0}}-U, \quad \frac{1}{\chi_{\mathrm{st}}}=\frac{1}{\chi_{\mathrm{st}}^{0}}-U,
$$

where $\chi_{m}^{0}$ and $\chi_{\mathrm{st}}^{0}$ are the respective susceptibilities of the noninteracting system. The random phase approximation for these susceptibilities is equivalent to the Hartree-Fock scheme described above. Therefore we may ask whether we obtain the IEM in the limit $U \ll t$ (where $m, m_{\text {st }} \ll 1$ ). The answer is not straightforward since the prefactors in the expansion (14) depend, for example, on the lattice structure. On bipartite lattices, as discussed here, they diverge for $T \rightarrow 0$. Thus an expansion of the free energy in powers of $m_{\mathrm{st}}$

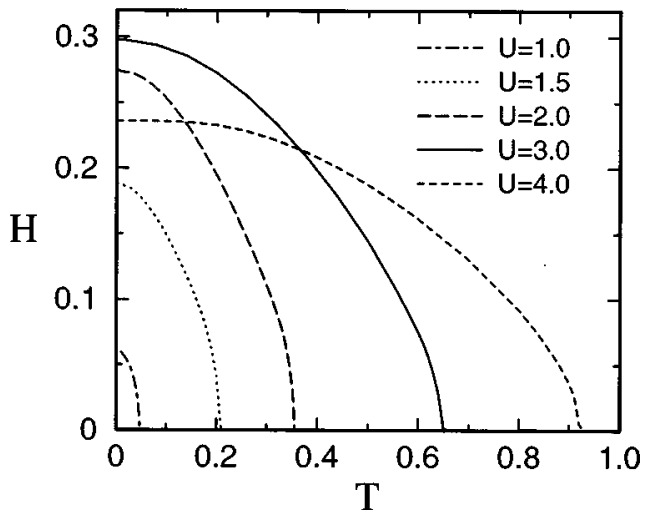

FIG. 3. $H-T$ phase diagram for different values of $U$ in HartreeFock approximation. All phase transitions are of first order. Below the curves the antiferromagnetic phase is stable. and $m$ as assumed in the IEM Landau theory is not possible in general (for details see Appendix C).

\section{B. Strong coupling}

In the limit $U \gg t$ the Hubbard model at half filling $(n=1)$ is equivalent to an effective Heisenberg spin model,

$$
\hat{H}_{\mathrm{Heis}}=\frac{J}{2} \sum_{\mathrm{NN}} \hat{\mathbf{S}}_{i} \cdot \hat{\mathbf{S}}_{j}-2 H \sum_{i} \hat{S}_{i}^{z},
$$

where the antiferromagnetic exchange coupling is obtained in second order perturbation theory as $J=4 t^{2} / U$. Spin operators are defined as $\hat{S}_{i}^{z}=\frac{1}{2}\left(\hat{n}_{i \uparrow}-\hat{n}_{i \downarrow}\right), \hat{S}_{i}^{x}=\frac{1}{2}\left(\hat{c}_{i \uparrow}^{\dagger} \hat{c}_{i \downarrow}+\hat{c}_{i \downarrow}^{\dagger} \hat{c}_{i \uparrow}\right)$, and $\hat{S}_{i}^{y}=-(i / 2)\left(\hat{c}_{i \uparrow}^{\dagger} \hat{c}_{i \downarrow}-\hat{c}_{i \downarrow}^{\dagger} \hat{c}_{i \uparrow}\right)$. For this model the Weiss molecular field theory becomes exact in $d=\infty$ yielding, under the constraint of uniaxial magnetization, the same results as for the Ising model. For Ising models metamagnetic phase transitions are well-studied. ${ }^{11}$ In the case of a purely antiferromagnetic nearest-neighbor coupling [see Eq. (16)] the phase transitions are of first order only at $T=0$, but of second order at all $T>0$. The transition line in the $H-T$ phase diagram has indeed the form shown in Fig. 1(a), but with a tricritical temperature of $T_{t}=0$. This behavior can be understood already within Weiss molecular field theory, where the ground state energy per site is

$$
E\left(m, m_{\mathrm{st}}\right)=\frac{J^{*}}{8}\left(m^{2}-m_{\mathrm{st}}^{2}\right)-H m
$$

with

$$
J^{*}=Z J=\frac{4 t^{* 2}}{U}
$$

Minimization with respect to $m$ and $m_{\text {st }}$ shows that the fully polarized antiferromagnet $\left(m_{\mathrm{st}}=1\right)$ has the lowest energy for $H<J^{*} / 4$, whereas the fully polarized ferromagnet $(m=1)$ is energetically favored for $H>J^{*} / 4$. Thus, by applying a magnetic field a first order transition is induced. At $H=J^{*} / 4$ the states are highly degenerated since all magnetic phases with $m+m_{\mathrm{st}}=1$ have the same energy. For $T>0$ this degeneracy is lifted by entropy which disfavors fully polarized phases. Therefore the first order transition at $T=0$ immediately becomes second order for $T>0$, i.e., $T_{t}=0$. Indeed, a tricritical point at a finite temperature is only obtained in the case of spin interactions which simultaneously favor both fully polarized antiferromagnetic and ferromagnetic configurations. In particular, adding a ferromagnetic interaction $J^{\prime}$ between next-nearest neighbors (NNN's) on a simple cubic lattice stabilizes both ferro- and antiferromagnetic order. ${ }^{11}$

While in the case of effective spin models a ferromagnetic NNN coupling term is introduced ad hoc, simply to obtain the first order phase transition, this term naturally arises if we expand the strong coupling perturbation series of the Hubbard model to $O\left(t^{4} / U^{3}\right)$. However, besides this $J^{\prime}$ term there also appear additional four-spin terms. For the hypercubic lattice the effective Hamiltonian $H_{\text {eff }}$ reads ${ }^{47}$

$$
\begin{aligned}
\hat{H}_{\mathrm{eff}}= & -\frac{J}{4} \sum_{N N} \hat{Q}_{i j}+\frac{J^{\prime}}{4} \sum_{i, \tau^{\prime} \neq \pm \tau} \hat{Q}_{i+\tau^{\prime}, i+\tau} \\
& +\frac{t^{4}}{U^{3}} A \sum_{\{\square\}}\left(\hat{Q}_{12} \hat{Q}_{34}+\hat{Q}_{14} \hat{Q}_{23}-\hat{Q}_{13} \hat{Q}_{24}\right),
\end{aligned}
$$




$$
\begin{gathered}
J=4\left(\frac{t^{2}}{U}+B \frac{Z t^{4}}{U^{3}}\right), \\
J^{\prime}=4 \frac{t^{4}}{U^{3}} C .
\end{gathered}
$$

Here $\tau$ and $\tau^{\prime}$ are lattice vectors connecting a site to its $Z$ neighbors, and $\square$ represents a plaquette. Each plaquette is counted only once: the four sites $\{1,2,3,4\}$ represent its four corners in clockwise or anticlockwise order. The constants $A, B$, and $C$ depend on the lattice, and the Hermitian operators $\hat{Q}_{i j}$ are defined as

$$
\hat{Q}_{i j}=-2\left(\hat{\mathbf{S}}_{i} \cdot \hat{\mathbf{S}}_{j}-\frac{1}{4}\right) .
$$

The plaquette contribution competes with the ferromagnetic NNN term $\left(J^{\prime}\right)$ and drives the system to second order phase transitions. For the hypercubic lattice the plaquette contribution is stronger than the ferromagnetic NNN term, yielding second order phase transitions even for $T=0$. The same is true for the Bethe lattice where, in fact, $J^{\prime}<0$ (for details see Appendix D). Thus in strong coupling perturbation theory the metamagnetic phase transition is of second order even at $T=0$.

\section{Intermediate coupling}

The perturbation analysis described above demonstrates that the order of the metamagnetic phase transition depends on the Coulomb interaction $U$ in a delicate way. For small $U$ the phase transition is purely of first order and for large $U$ of second order. Apparently, the tri- or multicritical point linking these two regimes must be found at intermediate coupling. In this important, nonperturbative regime quantum Monte Carlo techniques are employed to solve the problem numerically without any further approximation. ${ }^{24}$ The results for the magnetization $m(H)$ and the staggered magnetization $m_{\mathrm{st}}(H)$ are shown in Fig. 4 for $U=2$. Below the Néel temperature a metamagnetic behavior is clearly seen: for small magnetic fields the magnetization is exponentially suppressed with temperature. Then, towards the metamagnetic phase transition, the susceptibility increases drastically and becomes maximal at the critical field $H_{c}$. Second order phase transitions are observed for $1 / 14 \leqslant T \leqslant T_{N}=0.114$ \pm 0.006 , whereas the transition is of first order at lower temperatures, i.e., $T \leqslant 1 / 16$. At the phase transition the order parameter, i.e., the staggered magnetization, vanishes. From the curve $m_{\mathrm{st}}(H)$ the critical field $H_{c}$ and also the order of the phase transition is determined by a square root fit for second order transitions and by the mean of the hysteresis for first order transitions.

Using these values of $H_{c}$ the phase diagram, for different values of $U$ is constructed (Fig. 5). The case $U=4$ (= bandwidth) and half filling, Fig. 5(a), was already discussed in Ref. $24 .{ }^{43}$ It is shown here to illuminate the changes occurring under variation of $U$. This phase diagram shows both first order (for $T<1 / 16$ ) and second order phase transitions (for $1 / 8<T<T_{N} \approx 0.2$ ). At intermediate temperatures, i.e., $1 / 16<T<1 / 8$, the field dependence is rather more

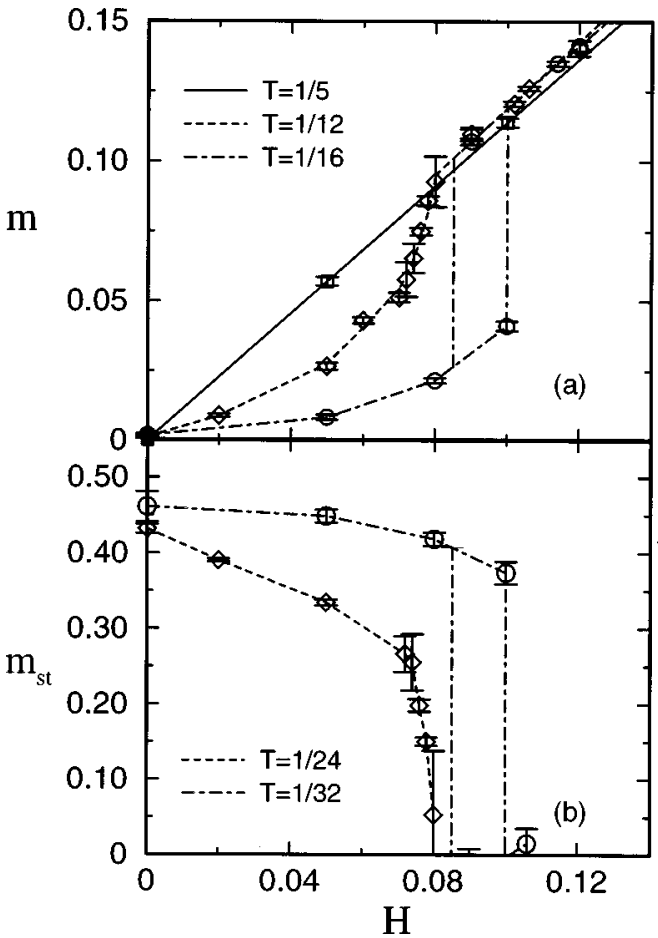

FIG. 4. QMC results, including error bars, (a) for the magnetization $m(H)$ as obtained for the $d=\infty$ Hubbard model with easy axis at half filling and $U=2$ (= half band width) for different temperatures. (b) Staggered magnetization $m_{\mathrm{st}}(H)$ for the two temperatures below $T_{N}$.

complicated. ${ }^{24}$ While the error bars do not permit an unambiguous interpretation it seems that the order parameter decreases by two consecutive transitions: the first oneseparating an $\mathrm{AF}$ phase with $m \approx 0\left(\mathrm{AF}_{\mathrm{I}}\right)$ and an $\mathrm{AF}$ phase

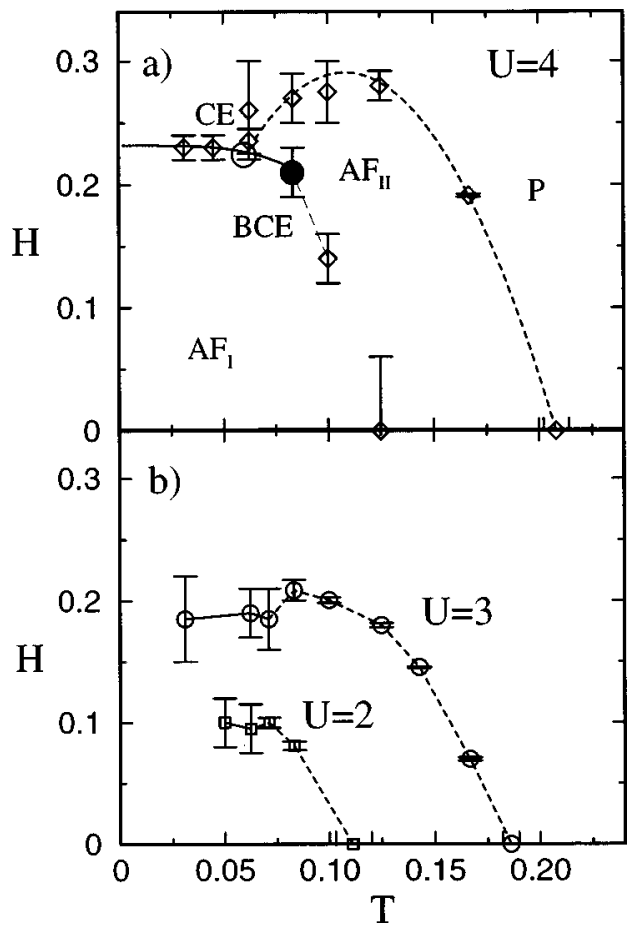

FIG. 5. H-T phase diagram for the $d=\infty$ Hubbard model with easy axis at half filling as constructed from the QMC results for $m(H)$ and $m_{\mathrm{st}}(H)$, (a) $U=4$, (b) $U=2,3$. Second order phase transitions are indicated by dashed lines, first order transitions by solid lines. Curves are guides to the eye only. 


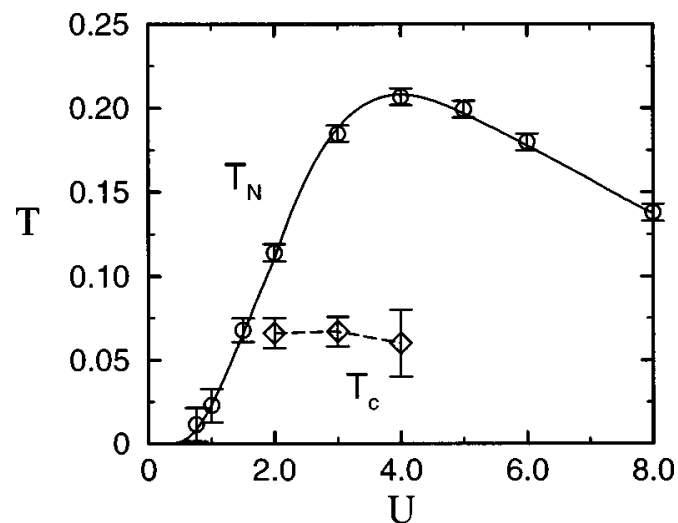

FIG. 6. Néel temperature $T_{N}$ (circles) and (tri-)critical temperature $T_{c}$ (diamonds) vs $U$. Above $T_{N}$ the system is paramagnetic. In an external magnetic field the order parameter vanishes in a second order metamagnetic phase transition for $T_{c}<T<T_{N}$ and in a first order transition for $T<T_{c}$, respectively.

with $m>0\left(\mathrm{AF}_{\mathrm{II}}\right)$-is of first order or corresponds to an anomaly, and the second one, at higher fields, is of second order. Taken together the results seem to correspond to the scenario of Fig. 1(b). For $U=2$ phase transitions of first order are found for $T \leqslant 1 / 16$ and of second order for $1 / 14 \leqslant T<T_{N}=0.114 \pm 0.006$ [see Fig. 5(b)]. Here, the temperature regime with two consecutive transitions, as obtained for $U=4$, has disappeared or has become very small: the scenario is similar to Fig. 1(a). The phase diagram for $U=3$, also displayed in Fig. 5(b), shows some features of the phase diagram in Fig. 1(b) or $U=4$, respectively. In particular one observes a maximum in the second order phase transition line and different slopes for the second and first order line at the crossover point. However, the numerical data do not indicate the existence of two consecutive transitions. Thus this phase diagram lies in between the scenarios depicted in Figs. 1(a) and 1(b).

To study the influence of the interaction $U$ qualitatively and quantitatively, it would be desirable to calculate $H-T$ phase diagrams at even larger values of $U$. Unfortunately the quantum Monte Carlo approach fails in this case due to the problem of "sticking" as mentioned in Sec. III. Therefore, we concentrate on the crossover from the intermediate coupling regime with first and second order transitions to the weak coupling regime with first order transitions only. The results for half filling are collected in Fig. 6 showing the $U$ dependence of two transition temperatures: the top curve is the Néel temperature $T_{N}$ at $H=0$ (taken from Ref. 45). The lower curve corresponds to the temperature $T_{c}$ where the second order phase transition line terminates, i.e., it represents either the tricritical or the critical temperature of Fig. 1. For temperatures below $T_{c}$ a first order metamagnetic phase transition is observed in an external magnetic field. Figure 6 reveals the crossover from intermediate coupling with first and second order phase transitions to weak coupling with first order transitions only: as $U$ decreases the regime with second order phase transitions $\left(T_{c}<T<T_{N}\right)$ shrinks, while the temperature regime for first order transitions remains nearly unchanged up to $U \approx 2$.

\section{RESULTS AWAY FROM HALF FILLING}

In the preceding sections metamagnetic transitions were investigated in the case of half filling. Beyond half filling the

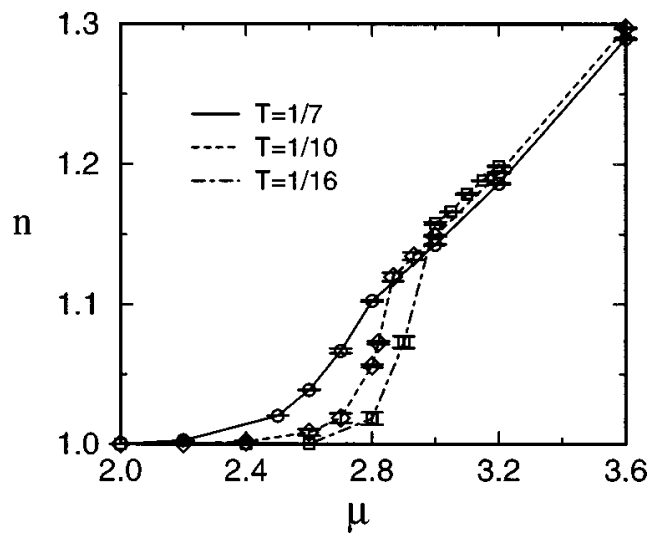

FIG. 7. Particle number $n$ vs chemical potential $\mu$ as calculated by grand canonical QMC simulations in the antiferromagnetic phase for $U=4$.

commensurate antiferromagnetic phase remains stable in the parameter regime under consideration $(\delta=|1-n| \leqslant 0.075$, $T \geqslant 1 / 32$ ). Incommensurate spin density waves become stable only in a small density regime at lower temperatures. ${ }^{48}$ Another possible instability of the antiferromagnetic phase away from half filling is phase separation, found within the Hartree-Fock approximation and in second order perturbation theory at constant order parameter at $T=0 .{ }^{49}$ However, at least for $T \geqslant 1 / 16$ we do not observe phase separation since the electronic compressibility $\kappa_{e}=\partial n / \partial \mu$ is finite and positive (see Fig. 7).

Upon doping the magnetization curve changes considerably and hardly indicates the existence of a metamagnetic phase transition (Fig. 8). This is due to the fact that in the metallic phase there is no longer a "Slater gap" at the Fermi energy; therefore the homogeneous susceptibility is not as strongly affected by the antiferromagnetic order as at half filling. The phase transition is, however, clearly seen in $m_{\mathrm{st}}(H)$. From the $m_{\mathrm{st}}$ vs $H$ curve the phase diagram (Fig. 9) is constructed. The metamagnetic phase transition line is found at lower temperatures and fields compared to half filling.

Associated with the metamagnetic phase transition is a change of the electrical resistivity. To study this important effect we calculated the $H$ dependence of the electronic compressibility $\kappa_{e}$. This quantity indicates whether the system is metallic or insulating. For an insulator $\kappa_{e}$ vanishes for $T=0$ and is exponentially small for temperatures lower than the antiferromagnetic gap. In a Fermi liquid, on the other hand, $\kappa_{e}(T=0)$ is finite since it is proportional to the density of states at the Fermi level and hence proportional to the Drude conductivity.

The results for $\kappa_{e}$ as a function of magnetic field $H$ at $U=2$ are shown in Fig. 10. At half filling, $\delta=0$, the compressibility is seen to increase with $H$. This effect is particular pronounced at low temperatures $(T=1 / 25)$ where $\kappa_{e}$ is essentially zero at low fields and rises to $\kappa_{e} \approx 0.3$ above the critical field, indicated by an arrow. Hence the metamagnetic phase transition is a transition from an antiferromagnetic insulator to a metal with homogeneous magnetization. At higher temperatures, $T=1 / 14$, the compressibility is always finite due to thermal excitations. We note that at $U=4$, when the electrons are essentially localized, $\kappa_{e}$ remains small 


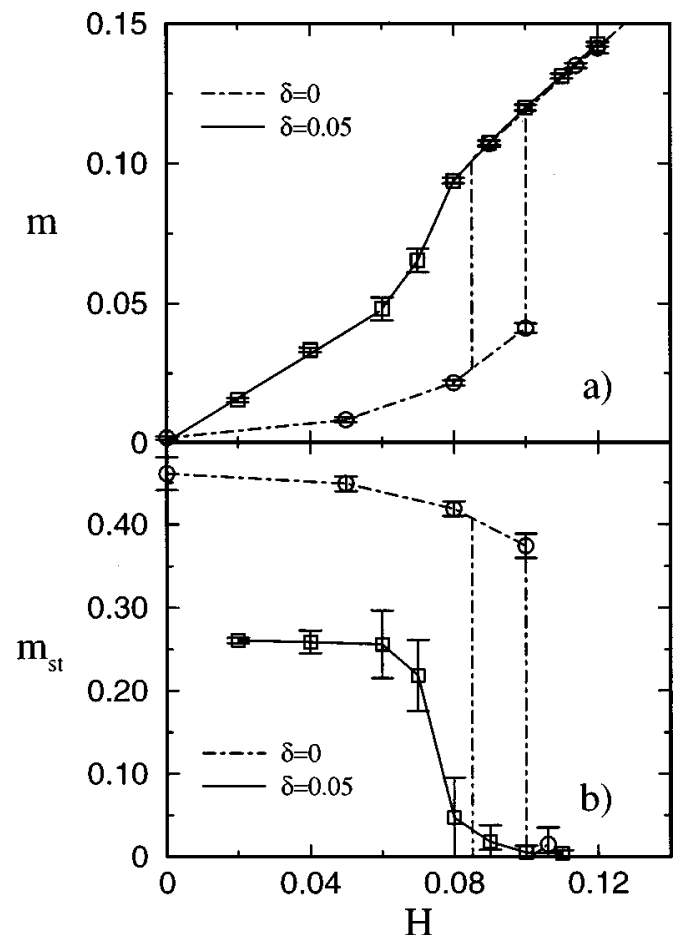

FIG. 8. Change of (a) the magnetization $m(H)$, and (b) the staggered magnetization $m_{\mathrm{st}}(H)$ with doping for $U=2$ at $T=1 / 16$.

$\left(0<\kappa_{e}<0.03\right.$ at $T=1 / 8$; not shown in Fig. 10) even above the critical field, indicating an insulator-to-insulator transition. The situation is very different at finite doping $(\delta=0.05)$. Here Fig. 10 shows that $\kappa_{e}$ decreases with $H$ by approximately $50 \%$ as the system goes through the metamagnetic transition from an antiferromagnetic metal to a metal with homogeneous magnetization.

\section{DISCUSSION}

Metamagnetism in strongly anisotropic antiferromagnets has been of interest to various communities in classical statistical mechanics for a long time already. In our paper we showed that this phenomenon can be derived from a model

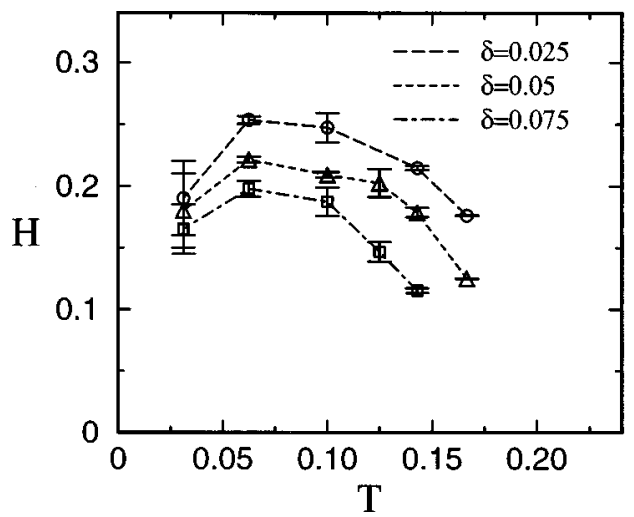

FIG. 9. Change of the $H-T$ phase diagram with doping $\delta$ for $U=4$. QMC data are shown for $\delta=0.025,0.05$, and 0.075. [For $\delta=0$ see Fig. 5(a).] Second order phase transitions are indicated by dashed lines, first order transitions by solid lines. For $T=1 / 32$ the numerical error does not permit the determination of the order of the phase transition unambiguously.

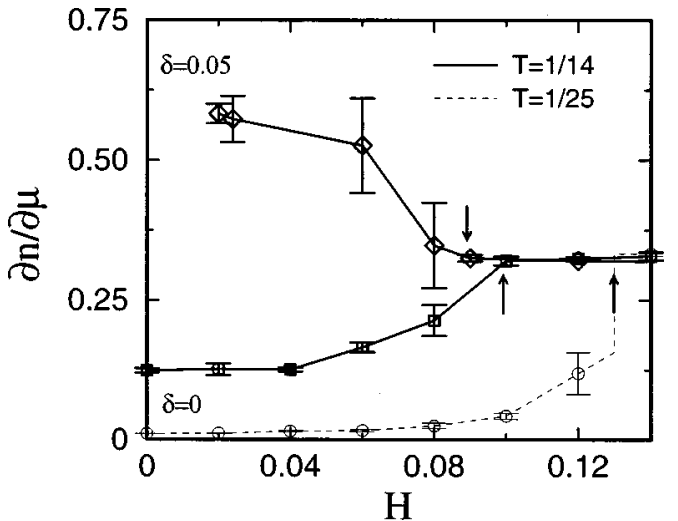

FIG. 10. Field dependence of the electronic compressibility $\kappa_{e}=\partial n / \partial \mu$ for $U=2$ at $\delta=0 \quad(T=1 / 14,1 / 25)$ and $\delta=0.05$ $(T=1 / 14)$. The arrows mark the respective critical fields for the metamagnetic phase transition.

of itinerant electrons, the Hubbard model with easy axis. This approach is fundamentally different from previous investigations since we identified and explicitly evaluated the simplest electronic, i.e., fully quantum mechanical, correlation model that is able to explain the conditions for metamagnetism. For this electronic model we employ the dynamical mean-field theory and show unambiguously that at intermediate coupling the phase transition is of first order at low temperatures and of second order near the Néel temperature, i.e., the order of the phase transition changes.

Our approach allows us to describe a broad range of qualitatively different metamagnets within a single model. While at present this simple model does not permit any quantitative calculation of material properties it does describe itinerant and localized, metallic, and insulating metamagnets and the crossover between them. This crossover is related to two fundamental experimental parameters, i.e., pressure (related to $U / t$ which decreases with pressure) and doping.

At half filling the Coulomb interaction leads to a crossover from a band insulator to an insulator with localized moments. Thereby the phase transition changes from first order for the bandlike metamagnet to second order for the localized one. Only at intermediate couplings are both first and second order phase transitions observed as found in experiment. The $H$-T phase diagram obtained for an intermediate Coulomb interaction ( $U=4=$ bandwidth) is strikingly similar to that of $\mathrm{FeBr}_{2}$ (Refs. 17-20 and 50) or the Ising model with weak ferromagnetic interaction. ${ }^{12,15}$

We note that in these insulating systems the applicability of a theory which becomes exact in the limit of a large coordination number is justified by the fact that the AF superexchange involves 20 equivalent sites in the two neighboring iron planes. ${ }^{51}$ At smaller values of the Coulomb interaction $(U=2)$ the temperature regime with second order transitions shrinks and the two step phase transition becomes less pronounced, reproducing the scenario of Fig. 1(a), as observed e.g., in $\mathrm{FeCl}_{2}$.

The calculations off half filling allow us to investigate the properties of metallic metamagnets, such as the uraniumbased mixed systems, ${ }^{5,6}$ for which a theory in terms of a correlated electron model is mandatory. In contrast to the insulating case, the metamagnetic phase transition in the me- 
tallic system is hardly visible in the magnetization curve. This is because there is no longer a gap at the Fermi energy. Quite generally, the critical temperatures and fields decrease upon doping.

The metamagnetic transition is accompanied by pronounced changes in the conductivity of the system. The Hubbard model with easy axis can qualitatively describe several scenarios:

(i) In the insulating, localized regime $(U \geqslant 4$ at half filling) a magnetic field causes a transition from an antiferromagnetic insulator to an insulator with homogeneous magnetization.

(ii) At lower $U$ values (e.g., $U=2$ ) at half filling an insulator-to-metal transition occurs at the magnetic field where the AF order disappears. Such a phenomenon is observed, for example, in the AF phase of $\mathrm{La}_{1-x} \mathrm{Ca}_{x} \mathrm{MnO}_{3}$, where the resistivity is found to change by several orders of magnitude. ${ }^{52}$ This is referred to as "colossal" magnetoresistance. We note that $\mathrm{La}_{1-x} \mathrm{Ca}_{x} \mathrm{MnO}_{3}$ shows no strong anisotropy. Therefore our approach can only describe the general features, in particular the existence of the insulator-to-metal transition.

(iii) Away from half filling a magnetic field induces a transition from a metallic antiferromagnet to a metal without staggered moment. Here the compressibility changes by less than an order of magnitude, e.g., about $50 \%$ at $U=2$, $\delta=0.05$. A similar effect is found in several strongly anisotropic antiferromagnets, both in multilayers and bulk intermetallic compounds such as UPdGe. ${ }^{6}$ In these systems the origin of this "giant" magnetoresistance is attributed to band structure effects and spin scattering. ${ }^{53}$ By contrast, our approach stresses the importance of genuine electronic correlation effects. More detailed investigations, including band degeneracy and spin-orbit interaction, may eventually provide even quantitative insight into these interesting and important phenomena.

\section{ACKNOWLEDGMENTS}

We acknowledge useful correspondence with N. Giordano, G. Lander, B. Lüthi, A. Ramirez, and W. Wolf, and are grateful to H. Capellmann, P. van Dongen, W. Metzner, H. Müller-Krumbhaar, J. Schlipf, F. Steglich, G. Stewart, and, in particular, to V. Dohm, W. Kleemann and W. Selke for very helpful discussions. In its initial stages this work was supported in part by the SFB 341 of the DFG and by a Grant from the ONR, N00014-93-1-0495.

\section{APPENDIX A: CALCULATION OF SUSCEPTIBILITIES FROM CORRELATION FUNCTIONS}

Quite generally susceptibilities can be obtained from the derivative of the order parameter $m_{x}$ with respect to the corresponding field $x$ :

$$
\chi_{x}=\frac{\partial m_{x}}{\partial x}=\frac{1}{2} T \sum_{\alpha, \sigma, n} f_{\alpha x}^{\sigma} \frac{\partial G_{\alpha n}^{\sigma}}{\partial x}
$$

with

$$
f_{\alpha x}^{\sigma}=\left\{\begin{array}{c}
\sigma \\
\alpha \sigma \\
1 \\
\alpha
\end{array}\right\} \quad \text { for } x=\left\{\begin{array}{c}
H \\
H_{\mathrm{st}} \\
\mu \\
\mu_{\mathrm{CDW}}
\end{array}\right\}
$$

Here $x=H$ and $x=H_{\text {st }}$ lead to the ferromagnetic and antiferromagnetic susceptibilities, $x=\mu$ to the electronic compressibility, and $x=\mu_{\mathrm{CDW}}$ to the charge density wave susceptibility. From the two self-consistency equations (2) and (3) one obtains two corresponding equations for the derivative of the Green function with respect to the variable $x$. The derivative of the functional integral (3) gives

$$
\frac{\partial G_{\alpha n}^{\sigma}}{\partial x}=T \sum_{\sigma^{\prime}, n^{\prime}} \Gamma_{n n^{\prime}, n^{\prime} n}^{\alpha \sigma \sigma^{\prime}} \gamma_{\alpha n^{\prime}}^{\sigma^{\prime} x},
$$

where $\Gamma$ is the local two-particle correlation function,

$$
\begin{aligned}
\Gamma_{n_{1} n_{1}^{\prime}, n_{2}^{\prime} n_{2}}^{\alpha \sigma \sigma^{\prime}}= & \left\langle\Psi_{\alpha n_{1}}^{\sigma} \Psi_{\alpha n_{2}}^{\sigma *} \Psi_{\alpha n_{1}^{\prime}}^{\sigma^{\prime}} \Psi_{\alpha n_{2}^{\prime}}^{\sigma^{\prime} *}\right\rangle-\delta_{\sigma \sigma^{\prime}}\left\langle\Psi_{\alpha n_{1}}^{\sigma} \Psi_{\alpha n_{2}}^{\sigma *}\right\rangle \\
& \times\left\langle\Psi_{\alpha n_{1}^{\prime}}^{\sigma} \Psi_{\alpha n_{2}^{\prime}}^{\sigma *}\right\rangle .
\end{aligned}
$$

The quantity $\gamma_{\alpha n}^{\sigma x}=(\partial / \partial x)\left\{\left(G_{\alpha n}^{\sigma}\right)^{-1}+\Sigma_{\alpha n}^{\sigma}\right\}$ in Eq. (A3) measures the response of the averaged medium to an infinitesimal change of the field $x$. This dynamical response function is determined by an integral equation in frequency space which does not explicitly depend on momentum. (Note that there are no convolutions in $\mathbf{k}$ space in the $d=\infty$ limit as is typical for a mean-field theory.) This property does not imply, however, that the response function $\gamma_{\alpha n}^{\sigma x}$ is local, too. It only indicates that $\gamma_{\alpha n}^{\sigma x}$ is diagonal in the momentum $\mathbf{k}$. Momentum dependence enters implicitly by the particular $\mathbf{k}$ dependence of the external field $[\mathbf{k}=0$ in the case of the compressibility or the ferromagnetic susceptibility, and $\mathbf{k}=(\pi, \ldots, \pi)$ for the staggered susceptibility].

In the presence of an external field the variables $z_{\alpha n}^{\sigma}$ in the Dyson equation (2) are replaced by $z_{\alpha n}^{\sigma}=i \omega_{n}+\mu+\sigma H$ $+\alpha \sigma H_{\mathrm{st}}+\alpha \mu_{\mathrm{CDW}}-\Sigma_{\alpha n}^{\sigma}$. The derivative of the Green function yields

$$
\begin{aligned}
\frac{\partial G_{\alpha n}^{\sigma}}{\partial x}= & \left\{\gamma_{\alpha n}^{\sigma x}+\frac{\partial G_{\alpha n}^{\sigma} / \partial x}{\left(G_{\alpha n}^{\sigma}\right)^{2}}-f_{\alpha x}^{\sigma}\right\} \zeta_{\alpha n}^{\sigma} \\
& +\left\{\gamma_{-\alpha n}^{\sigma x}+\frac{\partial G_{-\alpha n}^{\sigma} / \partial x}{\left(G_{-\alpha n}^{\sigma}\right)^{2}}-f_{-\alpha x}^{\sigma}\right\} \eta_{\alpha n}^{\sigma},
\end{aligned}
$$

with

$$
\begin{gathered}
\zeta_{\alpha n}^{\sigma}=\int_{-\infty}^{\infty} d \epsilon D(\epsilon)\left(z_{\alpha n}^{\sigma}-\epsilon^{2} / z_{-\alpha n}^{\sigma}\right)^{-2}, \\
\eta_{\alpha n}^{\sigma}=\int_{-\infty}^{\infty} d \epsilon D(\epsilon)\left(z_{\alpha n}^{\sigma}-\epsilon^{2} / z_{-\alpha n}^{\sigma}\right)^{-2} \epsilon^{2} /\left(z_{-\alpha n}^{\sigma}\right)^{2} .
\end{gathered}
$$

Since Eq. (A5) separates in Matsubara frequencies $n$ and spin $\sigma$ it can be easily solved for $\partial G / \partial x$, 


$$
\frac{\partial G_{\alpha n}^{\sigma}}{\partial x}=\sum_{\alpha^{\prime}} R_{n}^{\sigma \alpha \alpha^{\prime}}\left(f_{\alpha^{\prime} x}^{\sigma}-\gamma_{\alpha^{\prime} n}^{\sigma x}\right),
$$

with the $2 \times 2$ array in $\alpha \in\{A, B\}$ :

$$
\mathbf{R}_{n}^{\sigma}=-(\operatorname{det} \mathbf{D})^{-1} \mathbf{D}_{n}^{\sigma} \mathbf{T}_{n}^{\sigma},
$$

whereby $\mathbf{D}$ and $\mathbf{T}$ are defined as

$$
\begin{gathered}
\mathbf{D}_{n}^{\sigma=}=\left(\begin{array}{cc}
1-\frac{\zeta_{B n}^{\sigma}}{\left(G_{B n}^{\sigma}\right)^{2}} & \frac{\eta_{A n}^{\sigma}}{\left(G_{B n}^{\sigma}\right)^{2}} \\
\frac{\eta_{B n}^{\sigma}}{\left(G_{A n}^{\sigma}\right)^{2}} & 1-\frac{\zeta_{A n}^{\sigma}}{\left(G_{A n}^{\sigma}\right)^{2}}
\end{array}\right), \\
\mathbf{T}_{n}^{\sigma}=\left(\begin{array}{cc}
\zeta_{A n}^{\sigma} & \eta_{A n}^{\sigma} \\
\eta_{B n}^{\sigma} & \zeta_{B n}^{\sigma}
\end{array}\right)
\end{gathered}
$$

Now $\partial G / \partial x$ can be eliminated by setting Eq. (A3) equal to Eq. (A8), yielding

$$
\begin{aligned}
\sum_{\alpha^{\prime}} R_{n}^{\sigma \alpha \alpha^{\prime}} f_{\alpha^{\prime} x}^{\sigma}= & \sum_{\sigma^{\prime}, \alpha^{\prime}, n^{\prime}}\left\{\delta_{n n^{\prime}} \delta_{\sigma \sigma^{\prime}} R_{n}^{\sigma \alpha \alpha^{\prime}}+\delta_{\alpha \alpha^{\prime}} T \Gamma_{n n^{\prime}, n^{\prime} n}^{\alpha \sigma \sigma^{\prime}}\right\} \\
& \times \gamma_{\alpha^{\prime} n^{\prime}}^{\sigma^{\prime} x}
\end{aligned}
$$

From this equation we determine $\gamma$ by numerical inversion of a $4 \Lambda \times 4 \Lambda$ matrix. Knowing $\gamma$ we obtain $\partial G / \partial x$ via Eq. (A3) or Eq. (A8) and thus the susceptibility (A1).

\section{APPENDIX B: HARTREE-FOCK THEORY FOR $\mathbf{m}_{\mathrm{st}} \perp \mathbf{m}$}

Similar to the derivation of the Hartree-Fock equations for $\mathbf{m}\|\mathbf{H}\| \mathbf{m}_{\text {st }}$ (see Sec. IV A), we will now investigate the case with perpendicular orientation $\mathbf{m} \| \mathbf{H} \perp \mathbf{m}_{\mathrm{st}}$. The ansatz for the one-particle densities

$$
\left\langle\hat{n}_{i \in \alpha \sigma}\right\rangle=\frac{1}{2}(n+\sigma m),\left\langle\hat{c}_{i \in \alpha \sigma}^{\dagger} \hat{c}_{i \in \alpha-\sigma}\right\rangle=\frac{1}{2} \alpha m_{\mathrm{st}}
$$

yields in addition to the Hartree term a Fock term in the decoupling (8)

$$
\begin{aligned}
\hat{n}_{i \sigma} \hat{n}_{i-\sigma} \stackrel{\mathrm{HF}}{\rightarrow} & \hat{n}_{i \sigma}\left\langle\hat{n}_{i-\sigma}\right\rangle+\left\langle\hat{n}_{i \sigma}\right\rangle \hat{n}_{i-\sigma}-\left\langle\hat{n}_{i \sigma}\right\rangle\left\langle\hat{n}_{i-\sigma}\right\rangle \\
& -\hat{c}_{i \sigma}^{\dagger} \hat{c}_{i-\sigma}\left\langle\hat{c}_{i-\sigma}^{\dagger} \hat{c}_{i \sigma}\right\rangle-\left\langle\hat{c}_{i \sigma}^{\dagger} \hat{c}_{i-\sigma}\right\rangle \hat{c}_{i-\sigma}^{\dagger} \hat{c}_{i \sigma} \\
& +\left\langle\hat{c}_{i \sigma}^{\dagger} \hat{c}_{i-\sigma}\right\rangle\left\langle\hat{c}_{i-\sigma}^{\dagger} \hat{c}_{i \sigma}\right\rangle .
\end{aligned}
$$

With this ansatz one readily obtains the effective one-particle Hamiltonian

$$
\begin{aligned}
\hat{\mathrm{H}}_{\mathrm{HF}}= & \sum_{\mathrm{NN}, \sigma} t_{i j} \hat{c}_{i \sigma}^{\dagger} \hat{c}_{j \sigma}-\frac{1}{2} \frac{U}{4} \sum_{i, \sigma}\left(n^{2}-m^{2}-m_{\mathrm{st}}^{2}\right) \\
& +\sum_{\alpha, i \in \alpha, \sigma} \frac{U}{2}(n-\sigma m) \hat{n}_{i \sigma}-\frac{U}{2} \alpha m_{\mathrm{st}} \hat{c}_{i \sigma}^{\dagger} \hat{c}_{i-\sigma} \\
& -(\mu+\sigma H) \hat{n}_{i \sigma} .
\end{aligned}
$$

Diagonalizing this Hamiltonian yields the one-particle energies,

$$
\begin{aligned}
\tilde{\epsilon}_{\sigma}= & \operatorname{sgn}\left(\epsilon-\sigma\left\{\frac{U}{2} m+H\right\}\right) \\
& \times \sqrt{\left(\frac{U}{2} m_{\mathrm{st}}\right)^{2}+\left(\epsilon-\sigma\left\{\frac{U}{2} m+H\right\}\right)^{2}} .
\end{aligned}
$$

From these energies the free energy $\Omega$ is calculated, and the minimization with respect to $m$ and $m_{\text {st }}$ leads to the following Hartree-Fock self-consistency equations:

$$
\begin{aligned}
m_{\mathrm{st}} & =\frac{U}{2} \sum_{\sigma} \int d \epsilon N^{0}(\epsilon) \frac{-m_{\mathrm{st}}}{\widetilde{\epsilon}_{\sigma}} \frac{1}{1+e^{\beta\left(\widetilde{\epsilon}_{\sigma}-\tilde{\mu}\right)}}, \\
m & =\sum_{\sigma} \int d \epsilon N^{0}(\epsilon) \sigma \frac{\epsilon-\sigma\left(\frac{U}{2} m+H\right)}{\widetilde{\epsilon}_{\sigma}\left[1+e^{\beta\left(\tilde{\epsilon}_{\sigma}-\tilde{\mu}\right)}\right]} .
\end{aligned}
$$

As in Sec. IV A these Hartree-Fock equations are solved numerically.

\section{APPENDIX C: SERIES EXPANSION OF THE HARTREE-FOCK FREE ENERGY}

The itinerant electron metamagnetism theory of Moriya and Usami $^{23}$ can be derived from the Hartree-Fock approximation only if the free energy is analytic in $m$ and $m_{\mathrm{st}}$. Since in RPA the Hubbard interaction $U$ contributes to the free energy analytically [see Eqs. (14) and (15)] any nonanalytic behavior must be due to the kinetic energy. Its expansion in the order parameter $m_{\mathrm{st}}$ at $T=0$ is analyzed in this section.

To calculate the expansion in $m_{\mathrm{st}}$ a staggered magnetic field $H_{\text {st }}$ is introduced:

$$
\hat{\mathrm{H}}=\sum_{\mathrm{NN}, \sigma} t_{i j} \hat{c}_{i \sigma}^{\dagger} \hat{c}_{j \sigma}-H_{\mathrm{st}} \sum_{\alpha, i \in \alpha, \sigma} \alpha \sigma \hat{n}_{i \sigma}
$$

On $A-B$ lattices the one-particle energies for this Hamiltonian show a gap at $\epsilon=0$ with square root singularities at its edge,

$$
\widetilde{\epsilon}=\operatorname{sgn}(\epsilon) \sqrt{\epsilon^{2}+H_{\mathrm{st}}^{2}}
$$

We consider the half filled band, where the staggered magnetization $m_{\text {st }}$ is calculated from the one-particle energies. It shows the following asymptotic nonanalytic behavior for $H_{\mathrm{st}} \rightarrow 0$ :

$$
\begin{aligned}
& m_{\mathrm{st}}=\sum_{\sigma} \int_{-1}^{0} d \epsilon N^{0}(\epsilon) \frac{-H_{\mathrm{st}}}{\tilde{\epsilon}} \\
= & 2 N^{0}(0) H_{\mathrm{st}} \ln \left(1 / H_{\mathrm{st}}\right)+O\left(H_{\mathrm{st}}\right) .
\end{aligned}
$$

Similarly the asymptotic behavior of the energy (C1) is obtained as

$$
\begin{aligned}
\Delta E\left(m_{\mathrm{st}}\right) & =\sum_{\sigma} \int_{-1}^{0} d \epsilon N^{0}(\epsilon)(\tilde{\epsilon}-\epsilon) \\
& =-\frac{1}{2} H_{\mathrm{st}} m_{\mathrm{st}}+O\left(H_{\mathrm{st}}^{2}\right) .
\end{aligned}
$$


Subtracting the contribution due to $H_{\mathrm{st}}, \Delta E_{\mathrm{H}_{\mathrm{st}}}=-H_{\mathrm{st}} m_{\mathrm{st}}$, the asymptotic dependence of the kinetic energy on $m_{\text {st }}$ reads

$$
\Delta E_{\mathrm{kin}}\left(m_{\mathrm{st}}\right) \stackrel{H_{\mathrm{st}} \rightarrow 0}{\longrightarrow} \frac{1}{2} \frac{m_{\mathrm{st}}^{2}}{\ln \left(1 / m_{\mathrm{st}}\right)} .
$$

This shows that $\Delta E_{\mathrm{kin}}\left(m_{\mathrm{st}}\right)$ is nonanalytic in $m_{\mathrm{st}}$. Therefore the itinerant electron metamagnetism theory cannot be derived from the Hartree-Fock theory for the Hubbard model with an easy axis.

\section{APPENDIX D: THE METAMAGNETIC PHASE TRANSITION AT STRONG COUPLING}

In the limit of strong coupling and half filling the $O\left(t^{4} / U^{3}\right)$ perturbation theory yields the effective spin Hamiltonian (19). In the following, we study the metamagnetic phase transition, and especially the order of the transition, for this effective Hamiltonian. Restricting ourselves to solutions with mixtures of ferromagnetic $(m)$ and antiferromagnetic $\left(m_{\text {st }}\right)$ order, the ground state energy is a polynomial in $m$ and $m_{\text {st }}\left(t^{*} \equiv 1\right)$ :

$$
\begin{aligned}
E= & \frac{1}{2} \frac{1}{U} m^{2}-\frac{1}{2} \frac{1}{U} m_{\mathrm{st}}^{2}+\frac{1}{32} \frac{1}{U^{3}}\left\{16 B m^{2}-16 B m_{\mathrm{st}}^{2}+A m^{4}\right. \\
& -2 A m^{2} m_{\mathrm{st}}^{2}-2 A m^{2}+A m_{\mathrm{st}}^{4}+6 A m_{\mathrm{st}}^{2} \\
& \left.-16 C m^{2}-16 C m_{\mathrm{st}}^{2}\right\}-H m+\text { const. }
\end{aligned}
$$

One can see that the ferromagnetic next-nearest-neighbor interaction $C$ favors both saturated antiferromagnetism and saturated ferromagnetism rather than ferrimagnetic phases. By contrast the plaquette term $A$ has contributions that support the formation of a ferrimagnetic state. To obtain the ground state the energy must be minimized with respect to $m$ and $m_{\text {st }}$ under the constraints $|m| \leqslant 1$ and $\left|m_{\text {st }}\right| \leqslant 1-|m|$. Differentiation of $E$ with respect to $m_{\text {st }}$ shows that, for fixed $m$, $E$ has one maximum at $m_{\mathrm{st}}=0$ and two minima at

$$
m_{\mathrm{st}}= \pm \sqrt{\frac{8 U^{2}+A m^{2}-3 A+8 B+8 C}{A}} .
$$

For sufficiently strong coupling $U$ (e.g., $U>2$ in the case of the hypercubic lattice with $A=20, C=2$, and $B=4$ ) these minima are outside the constraint $\left|m_{\text {st }}\right| \leqslant 1-|m|$. Therefore $E$ becomes minimal at the border of the constraint, i.e., for $\left|m_{\text {st }}\right|=1-|m|$. Replacing $m_{\text {st }}$ by $1-m$ the minimization with respect to $m$ readily yields for the ground state,

$$
m=\left\{\begin{array}{c}
0 \text { for } H \leqslant \frac{1}{2} \frac{-A+2 C+2 U^{2}+2 B}{U^{3}} \\
\frac{1 \text { for } H \geqslant \frac{-C+U^{2}+B}{U^{3}}}{A-2 C-2 U^{2}-2 B+2 H U^{3}} \text { else, } \\
m_{\mathrm{st}}=1-m .
\end{array}\right.
$$

This ground state solution for the effective spin Hamiltonian shows a second order metamagnetic phase transition for $A>4 C$. This is the case for the hypercubic lattice ( $A=20, C=2$ ) and for the Bethe lattice, where $A=0$ but $C=-1$, i.e., the next-nearest-neighbor coupling is antiferromagnetic. In conclusion, the strong coupling theory shows second order phase transition for all temperatures, even at $T=0$.
*Electronic address: held@physik.uni-augsburg.de

†Present address: Theoretische Physik III, Universitat Augsburg, D-86135 Augsburg, Germany.

${ }^{1}$ L. Néel, Ann. Phys. (Paris) 18, 5 (1932).

${ }^{2}$ L. D. Landau, Phys. Z. Sowjetunion 4, 675 (1933), reprinted in Collected Papers of L. D. Landau, edited by D. ter Haar (Pergamon, London, 1965), p. 73.

${ }^{3}$ J. Becquerel and J. van den Handel, J. Phys. Radium 10, 10 (1939).

${ }^{4}$ This name was apparently coined by H. A. Kramers; see J. Becquerel, in Le Magnétisme (Institut International de Cooperation Intellectuelle, CNRS, Paris, 1940), p. 97.

${ }^{5}$ For a review see E. Stryjewski and N. Giordano, Adv. Phys. 26, 487 (1977).

${ }^{6}$ V. Sechovský et al., J. Appl. Phys. 76, 6913 (1994).

${ }^{7}$ J. H. V. J. Brabers et al., Phys. Rev. B 50, 16410 (1994).

${ }^{8}$ V. Ivanov et al., J. Alloys Compd. 218, L19 (1995).

${ }^{9}$ For a review see B. Lüthi et al., J. Magn. Magn. Mater. 90\&91, 37 (1991).

${ }^{10}$ L. D. Landau, Phys. Z. Sowjetunion 11, 26 (1937), reprinted in Collected Papers of L. D. Landau, edited by D. ter Haar (Pergamon, London, 1965), p. 193.

${ }^{11}$ For a review see I. D. Lawrie and S. Sarbach, in Phase Transitions and Critical Phenomena, edited by C. Domb and J. L. Lebowitz (Academic Press, New York, 1984), Vol. 9, p. 1.
${ }^{12}$ J. M. Kincaid and E. G. D. Cohen, Phys. Lett. 50A, 317 (1974); Phys. Rep. 22, 57 (1975).

${ }^{13}$ H. J. Herrmann and D. P. Landau, Phys. Rev. B 48, 239 (1993).

${ }^{14}$ W. Selke and S. Dasgupta, J. Magn. Magn. Mater. 147, L245 (1995).

${ }^{15}$ W. Selke, Z. Phys. B 101, 145 (1996).

${ }^{16}$ M. Pleimling and W. Selke, Phys. Rev. B 56, 8855 (1997).

${ }^{17}$ A. R. Fert et al., J. Phys. Chem. Solids 34, 223 (1973); C. Vettier, H. L. Alberts, and D. Bloch, Phys. Rev. Lett. 31, 1414 (1973).

${ }^{18}$ M. M. P. de Azevedo et al., J. Magn. Magn. Mater. 140-144, 1557 (1995); J. Pelloth et al., Phys. Rev. B 52, 15372 (1995).

${ }^{19}$ H. A. Katori, K. Katsumata, and M. Katori, Phys. Rev. B 54, R9620 (1996).

${ }^{20}$ K. Katsumata, H. A. Katori, S. M. Shapiro, and G. Shirane, Phys. Rev. B 55, 11466 (1997).

${ }^{21}$ O. Petracic, Ch. Binek, and W. Kleemann, J. Appl. Phys. 81, 4145 (1997).

${ }^{22}$ E. P. Wohlfarth and P. Rhodes, Philos. Mag. 7, 1817 (1962).

${ }^{23}$ T. Moriya and K. Usami, Solid State Commun. 23, 935 (1977).

${ }^{24}$ K. Held, M. Ulmke, and D. Vollhardt, Mod. Phys. Lett. B 10, 203 (1996).

${ }^{25}$ J. Hubbard, Proc. R. Soc. London, Ser. A 276, 238 (1963); M. C. Gutzwiller, Phys. Rev. Lett. 10, 59 (1963); J. Kanamori, Prog. Theor. Phys. 30, 275 (1963)

${ }^{26}$ M. Takahashi, Prog. Theor. Phys. 42, 1098 (1969). 
${ }^{27}$ In addition to the spin-orbit interaction there is also the dipoledipole interaction between electronic spins. The latter is mainly responsible for the "shape anisotropy," i.e., the fact that the direction of the easy axis depends on the shape of the probe. An introduction into the origins and models of the magnetic anisotropy is given by P. Bruno, Physical Origins and Theoretical Models of Magnetic Anisotropy, in Magnetismus von Festkörpern und Grenzflächen. (Forschungszentrum Jülich, IFF Ferienkurs, 1993), p. 24.1; P. Bruno, Phys. Rev. B 39, 865 (1989).

${ }^{28}$ An attempt to treat this problem within the Stoner model was made by K. Kulakowski and B. Barbara, Trieste Report No. IC/90/282, 1990 (unpublished).

${ }^{29}$ This procedure is quite similar to assuming the existence of a lattice on which the Hubbard model is defined. The existence of a lattice and the correlation physics described by the Hubbard model are a priori quite unrelated, and hence we do not need to start on the level of interacting electrons in the continuum.

${ }^{30}$ W. Metzner and D. Vollhardt, Phys. Rev. Lett. 62, 324 (1989).

${ }^{31}$ D. Vollhardt, in Correlated Electron Systems, edited by V. J. Emery (World Scientific, Singapore, 1993), p. 57.

${ }^{32}$ A. Georges, G. Kotliar, W. Krauth, and M. Rozenberg, Rev. Mod. Phys. 68, 13 (1996).

${ }^{33}$ V. Janiš, Z. Phys. B 83, 227 (1991).

${ }^{34}$ A. Georges and G. Kotliar, Phys. Rev. B 45, 6479 (1992).

${ }^{35}$ M. Jarrell, Phys. Rev. Lett. 69, 168 (1992).

${ }^{36}$ For a review see G. Kotliar, in Strongly Correlated Electronic Materials, edited by K. S. Bedell et al. (Addison-Wesley, Reading, 1994), p. 141.

${ }^{37}$ F. Gebhard, The Mott Metal-Insulator Transition, Springer Tracts in Modern Physics (Springer, Heidelberg, 1997), Vol. 137.

${ }^{38}$ For a review see Th. Pruschke, M. Jarrell, and J. K. Freericks, Adv. Phys. 44, 187 (1995).
${ }^{39}$ L. Laloux, A. Georges, and W. Krauth, Phys. Rev. B 50, 3092 (1994).

${ }^{40}$ T. Saso and T. Hayashi, J. Phys. Soc. Jpn. 63, 401 (1994).

${ }^{41}$ A. Giesekus and U. Brandt, Phys. Rev. B 48, 10311 (1993).

${ }^{42}$ This transition was also studied within a slave-boson mean-field theory. P. Korbel, J. Spałek, W. Wójcik, and M. Acquarone, Phys. Rev. B 52, R2213 (1995).

${ }^{43}$ Note that the energy scale has changed by a factor of two compared to Ref. 24 where $t^{*}=1 / 2$ was chosen. To compare the results, all numerical values of quantities with dimension energy (inverse energy) in Ref. 24 have to be multiplied (divided) by a factor of two.

${ }^{44}$ J. E. Hirsch and R. M. Fye, Phys. Rev. Lett. 56, 2521 (1986).

${ }^{45}$ For more details concerning the implementation see M. Ulmke, V. Janiš, and D. Vollhardt, Phys. Rev. B 51, 10411 (1995), all calculations were performed on a Cray YMP of the Forschungszentrum Jülich.

${ }^{46}$ It can be shown that the Hartree-Fock results remain qualitatively correct within second order perturbation theory at fixed order parameter: P. G. J. van Dongen, Phys. Rev. B 50, 14016 (1994).

${ }^{47}$ M. Takahashi, J. Phys. C 10, 1289 (1977); P. G. J. van Dongen, Phys. Rev. B 49, 7904 (1994).

${ }^{48}$ J. K. Freericks and M. Jarrell, Phys. Rev. Lett. 74, 186 (1995).

${ }^{49}$ P. G. J. van Dongen, Phys. Rev. Lett. 74, 182 (1995).

${ }^{50}$ Note that the mixed-phase region in the phase diagram of Ref. 39 disappears when instead of the applied field $H_{a}$ the internal field $H$ is plotted vs $T$.

${ }^{51}$ L. Hernández, H. T. Diep, and D. Bertrand, Europhys. Lett. 21, 711 (1993).

${ }^{52}$ A. P. Ramirez, J. Phys. Condens. Matter 9, 8171 (1997).

${ }^{53}$ P. M. Levy and S. Zhang, J. Magn. Magn. Mater. 151, 315 (1995). 